\title{
A new variable stiffness suspension system: passive case
}

\author{
O. M. Anubi, D. R. Patel, and C. D. Crane III \\ Center for Intelligent Machines and Robotics, Department of Mechanical and Aerospace Engineering, \\ University of Florida, Gainesville, Florida, USA \\ Correspondence to: O. M. Anubi (anubimoses@gmail.com)
}

Received: 15 November 2012 - Accepted: 15 January 2013 - Published: 26 February 2013

\begin{abstract}
This paper presents the design, analysis, and experimental validation of the passive case of a variable stiffness suspension system. The central concept is based on a recently designed variable stiffness mechanism. It consists of a horizontal control strut and a vertical strut. The main idea is to vary the load transfer ratio by moving the location of the point of attachment of the vertical strut to the car body. This movement is controlled passively using the horizontal strut. The system is analyzed using an $\mathcal{L}_{2}$-gain analysis based on the concept of energy dissipation. The analyses, simulation, and experimental results show that the variable stiffness suspension achieves better performance than the constant stiffness counterpart. The performance criteria used are; ride comfort, characterized by the car body acceleration, suspension deflection, and road holding, characterized by tire deflection.
\end{abstract}

\section{Introduction}

Improvements over passive suspension designs is an active area of research, as documented by the works of Alkhatib et al. (2004); Williams (1997); Butsuen (1989); Tseng and Hedrick (1994); Valasek and Kortum (1998, 2001); Karnopp et al. (1974); Karnopp (1983); Karnopp and Heess (1991); Evers et al. (2011); van der Knaap et al. (2008). Past approaches utilize one of three techniques (Ashfak et al., 2011); adaptive (Fialho and Balas, 2002), semi-active (Do et al., 2010; Butsuen, 1989) or fully active suspension (Williams et al., 1993). An adaptive suspension utilizes a passive spring and an adjustable damper with slow response to improve the control of ride comfort and road holding. A semi-active suspension is similar, except that the adjustable damper has a faster response and the damping force is controlled in realtime. A fully active suspension replaces the damper with a hydraulic actuator, or other types of actuators such as electromagnetic actuators, which can achieve optimum vehicle control, but at the cost of design complexity, expense, etc. The fully active suspension is also not fail-safe in the sense that performance degradation results whenever the control fails, which may be due to either mechanical, electrical, or software failures. Recently, research in semi-active suspensions has continued to advance with respect to capabilities, narrowing the gap between semi-active and fully active suspension systems. Today, semi-active suspensions (e.g. using Magneto-Rheological (MR) (Ashfak et al., 2011), ElectroRheological (ER) (Sung et al., 2007) etc.) are widely used in the automobile industry due to their small weight and volume, as well as low energy consumption compared to purely active suspension systems.

However, most semi-active suspension systems are designed to only vary the damping coefficient of the shock absorber while keeping the stiffness constant. Meanwhile, in suspension optimization, both the damping coefficient and the spring rate of the suspension elements are usually used as optimization arguments. Therefore, a semi-active suspension system that varies both the stiffness and damping of the suspension element could provide more flexibility in balancing competing design objectives. Suspension designs that exhibit variable stiffness phenomenon are few in literature considering the vast amount of researches that has been done on semi-active suspension designs. van der Knaap (1989); Venhovens and van der Knaap (1995); Evers et al. (2011) designed a variable geometry actuator for vehicle suspension 
called the Delft active suspension (DAS). Although, the intention of the design was not to vary the stiffness of the suspension system, the design used a variable geometry concept to vary the suspension force by effectively changing the stiffness of the suspension system. The basic idea behind the DAS concept is based on a wishbone which can be rotated over an angle and is connected to a pretensioned spring at a variable location. The spring pretension generates an effective actuator force, which can be manipulated by changing the position. This was achieved using an electric motor. Jerz (1971) invented a variable stiffness suspension system which includes two springs connected in series. One of the springs is stiffer than the other. Under normal load conditions, the softer spring is responsible for keeping a good ride comfort. Upon the imposition of heavier load forces, the vehicle is supported more stiffly and primarily by the stronger spring. Conversion between the two conditions was done automatically by engagement under heavy load conditions of a pair of stop shoulders acting to limit the compression of the light spring. Similarly, upon excessive extension of the springs, an additional set of stop shoulders are engaged automatically to limit the amount of extension of the softer spring and causes the stiffer spring to resist further extension. Kobori proposed a variable stiffness system to suppress a buildings' responses to earthquakes (Kobori et al., 1993). The aim was to achieve a non-stationary and no-resonant state during earthquakes. Youn and Hac used an air spring in a suspension system to vary the stiffness among three discrete values (Youn and Hac, 1995). Liu et al. (2008) proposed a suspension system which uses two controllable dampers and two constant springs to achieve variable stiffness and damping. A Voigt element and a spring in series are used to control system stiffness. The Voigt element is comprised of a controllable damper and a constant spring. The equivalent stiffness of the whole system is changed by controlling the damper in the Voigt element.

This paper presents the design and analysis of the passive case of a variable stiffness suspension system. The variation of stiffness concept used in this chapter uses the "reciprocal actuation" (Anubi et al., 2010) to effectively transfer energy between a vertical traditional strut and a horizontal oscillating control mass, thereby improving the energy dissipation of the overall suspension. Due to the relatively fewer number of moving parts, the concept can easily be incorporated into existing traditional front and rear suspension designs. An implementation with a double wishbone is shown in this paper. The rest of this paper is organized as follows. In Sect. 2, the variable stiffness concept is described, and the variable stiffness suspension system introduced. A detailed analysis of the system is presented in Sect. 3. Section 3.3 describes the analysis of the passive case. Experimental results are given in Sect. 4. Time domain and frequency domain simulation results are presented in Sect. 5. The conclusion follows in Sect. 6.

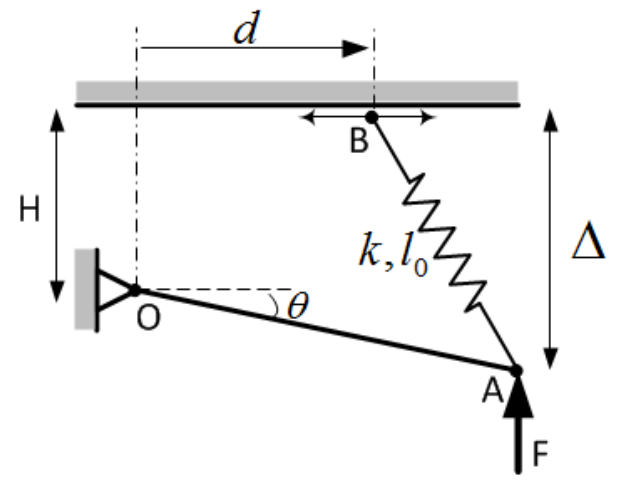

Figure 1. Variable Stiffness Mechanism.

\section{System description}

This section gives a detailed description of the variable stiffness concept, the overall system, its incorporation in a vehicle suspension, and the resulting system dynamic model.

\subsection{Variable stiffness concept}

The variable stiffness mechanism concept is shown in Fig. 1. The Lever arm OA, of length $L$, is pinned at a fixed point $\mathrm{O}$ and free to rotate about $\mathrm{O}$. The spring $\mathrm{AB}$ is pinned to the lever arm at $\mathrm{A}$ and is free to rotate about $\mathrm{A}$. The other end $\mathrm{B}$ of the spring is free to translate horizontally as shown by the double headed arrow. It is also free to rotate about point B. Without loss of generality, the external force $F$ is assumed to act vertically upwards at point A. $d$ is the horizontal distance of $\mathrm{B}$ from $\mathrm{O}$. The idea is to vary the overall stiffness of the system by letting $d$ vary passively under the influence of a horizontal spring-damper system (not shown in the figure). Let $k$ and $l_{0}$ be the spring constant and the free length of the spring $\mathrm{AB}$ respectively, and $\Delta$ the vertical displacement of the point $\mathrm{A}$. The overall free length $\Delta_{0}$ of the mechanism is defined as the value of $\Delta$ when no external force is acting on the mechanism.

\subsection{Mechanism description}

The suspension system considered is shown in Fig. 2. The schematic diagram is shown in Fig. 3. The model is composed of a quarter car body, wheel assembly, two springdamper systems, road disturbance, and lower and upper wishbones. The points $\mathrm{O}, \mathrm{A}$, and $\mathrm{B}$ are the same as shown in the variable stiffness mechanism of Fig. 1. The horizontal control force $u$ controls the position $d$ of the control mass $m_{d}$ which, in turn, controls the overall stiffness of the mechanism. The tire is modeled as a linear spring of spring constant $k_{\mathrm{t}}$. 


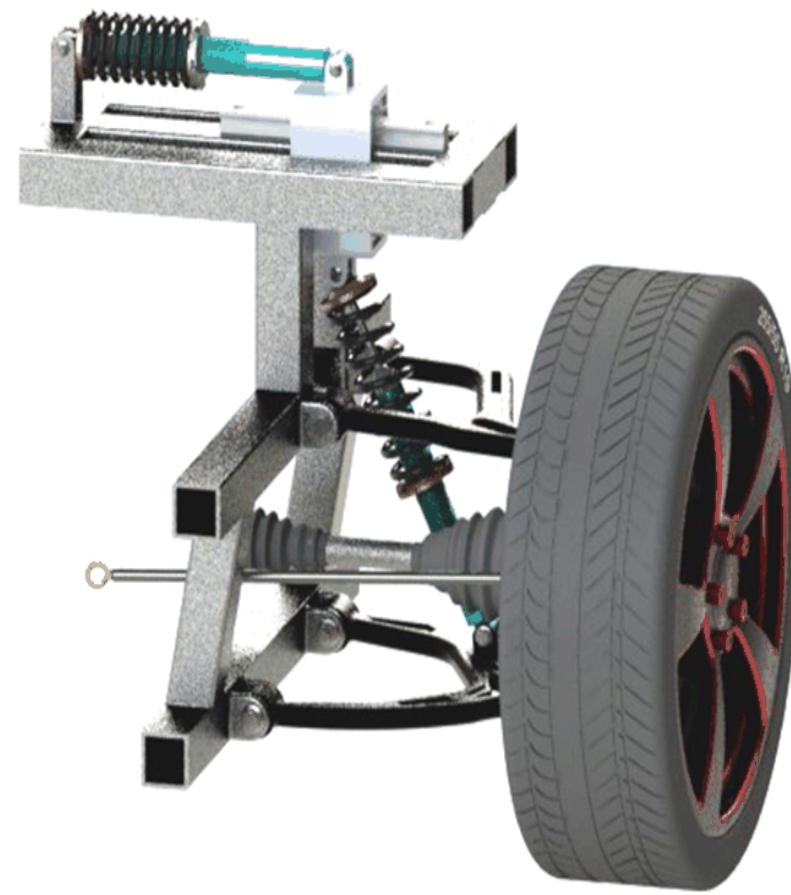

Figure 2. Variable Stiffness Suspension System.

The assumptions adopted in Fig. 3 are summarized as follows:

1. The lateral displacement of the sprung mass is neglected, i.e only the vertical displacement $y_{\mathrm{s}}$ is considered.

2. The wheel camber angle is zero at the equilibrium position and its variation is negligible throughout the system trajectory.

3. The springs and tire deflections are in the linear regions of their operating ranges.

\subsection{Equations of motion}

Let

$\boldsymbol{q}=\left[\begin{array}{c}y_{\mathrm{s}} \\ \theta \\ d\end{array}\right]$,

be defined as the generalized coordinates. The equations of motion, derived using Lagrange's method, are then given by

$$
\begin{array}{r}
\mathbf{M}(\theta) \ddot{\boldsymbol{q}}+\mathbf{C}(\theta, \dot{\theta})+\mathbf{B}(\theta) \dot{\boldsymbol{q}}-\mathbf{K}(\boldsymbol{q})+\mathbf{G}(\theta) \\
=\mathbf{e}_{3,3} u+\mathbf{W}_{\mathbf{d}}(\theta) \mathbf{d}_{r}
\end{array}
$$

where

$\mathbf{M}(\theta)=\left[\begin{array}{ccc}m_{\mathrm{s}}+m_{\mathrm{u}}+m_{d} & m_{\mathrm{u}} l_{D} \cos \theta & 0 \\ m_{\mathrm{u}} l_{D} \cos \theta & I_{\mathrm{c}}+m_{\mathrm{u}} l_{D}^{2} \cos ^{2} \theta & 0 \\ 0 & 0 & m_{d}\end{array}\right]$,

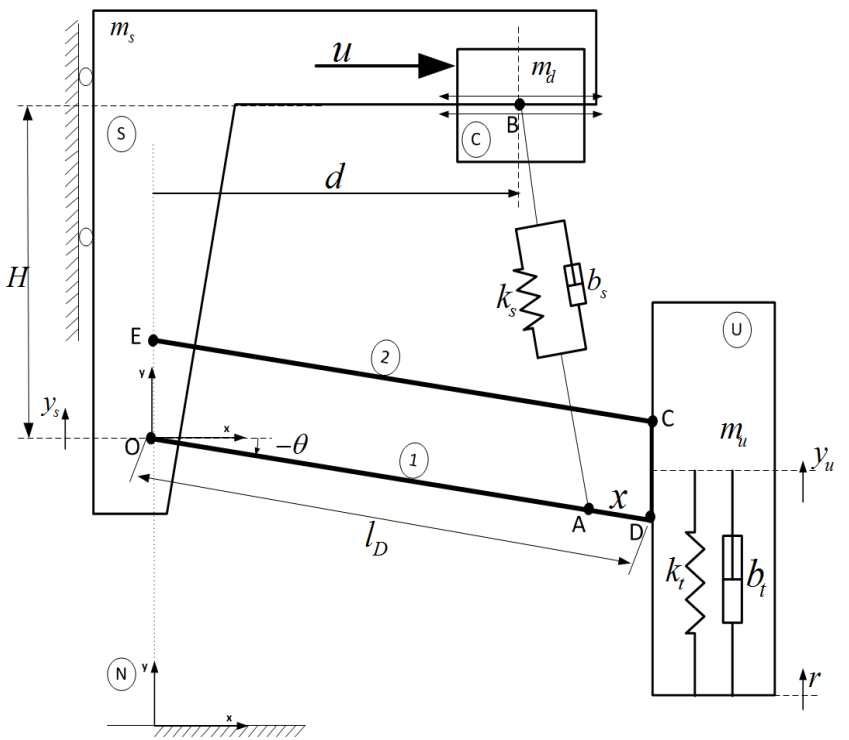

S: Sprung Mass (Chassis)

1: Lower Wishbone

2: Upper Wishbone

U: Unsprung Mass (Wheel Assembly)

C: Control Mass

$\mathrm{N}$ : Inertial Reference Frame

Figure 3. Quarter Car Model

$\mathbf{C}(\theta, \dot{\theta})=-m_{\mathrm{u}} l_{D} \dot{\theta}^{2} \sin \theta \boldsymbol{w}(\theta)$,

$\boldsymbol{w}(\theta)=\left[\begin{array}{c}1 \\ l_{D} \cos \theta \\ 0\end{array}\right]$,

$\mathbf{B}(\theta)=\left[\begin{array}{ccc}b_{\mathrm{t}} & b_{\mathrm{t}} l_{D} \cos \theta & 0 \\ b_{\mathrm{t}} l_{D} \cos \theta & b_{\mathrm{t}} l_{D}^{2} \cos ^{2} \theta+b_{\mathrm{s}} g_{\theta} & \frac{b_{\mathrm{s}}}{2} g_{d \theta} \\ 0 & \frac{b_{\mathrm{s}}}{2} g_{d \theta} & b_{\mathrm{s}} g_{d}\end{array}\right]$

$g_{d}(d, \theta)=\frac{\left(d-l_{A} \cos \theta\right)^{2}}{H^{2}+d^{2}+l_{A}^{2}-2 l_{A} d \cos \theta-2 H l_{A} \sin \theta}$,

$g_{d \theta}(d, \theta)=\frac{2 l_{A}\left(d-l_{A} \cos \theta\right)(d \sin \theta-H \cos \theta)}{H^{2}+d^{2}+l_{A}^{2}-2 l_{A} d \cos \theta-2 H l_{A} \sin \theta}$,

$g_{\theta}(d, \theta)=\frac{l_{A}^{2}(d \sin \theta-H \cos \theta)^{2}}{H^{2}+d^{2}+l_{A}^{2}-2 l_{A} d \cos \theta-2 H l_{A} \sin \theta}$,

$\mathbf{K}(\boldsymbol{q})=\left[\begin{array}{c}k_{\mathrm{t}}\left(\rho_{\mathrm{t}}-1\right)\left(y_{\mathrm{s}}+l_{D} \sin \theta\right) \\ k_{\mathrm{t}}\left(\rho_{\mathrm{t}}-1\right) l_{D} \cos \theta\left(y_{\mathrm{s}}+l_{D} \sin \theta\right) \\ k_{\mathrm{s}}\left(\rho_{\mathrm{s}}-1\right)\left(d-l_{A} \cos \theta\right)\end{array}\right]$

$+\left[\begin{array}{c}0 \\ k_{\mathrm{s}}\left(\rho_{\mathrm{s}}-1\right) l_{A}(d \sin \theta-H \cos \theta) \\ 0\end{array}\right]$, 


$$
\begin{aligned}
\mathbf{G}(\theta) & =\left[\begin{array}{c}
m_{\mathrm{s}}+m_{\mathrm{u}}+m_{d} \\
m_{\mathrm{u}} l_{D} \cos \theta \\
0
\end{array}\right] g, \\
\mathbf{W}_{\mathbf{d}}(\theta) & =\left[\begin{array}{cc}
k_{\mathrm{t}}\left(\rho_{\mathrm{t}}-1\right) & b_{\mathrm{t}} \\
k_{\mathrm{t}} l_{D}\left(\rho_{\mathrm{t}}-1\right) \cos \theta & b_{\mathrm{t}} l_{D} \cos \theta \\
0 & 0
\end{array}\right], \\
\mathbf{d}_{r} & =\left[\begin{array}{c}
r \\
\dot{r}
\end{array}\right] .
\end{aligned}
$$

$r(t)$ is the road displacement signal. It is a function of the road profile and the vehicle velocity. The terms $\rho_{\mathrm{s}}$ and $\rho_{\mathrm{t}}$ characterize the compression of the vertical strut and tire springs respectively. They are defined as the instantaneous length divided by its free length.

\section{Properties}

The following properties of the dynamics given in Eq. (2) are exploited in subsequent analyses:

1. The inertia matrix $\mathbf{M}(\theta)$ is symmetric, positive definite. Also, since each element of $\mathbf{M}(\theta)$ can be bounded below and above by positive constants, it follows that the eigenvalues, hence the singular values of $\mathbf{M}(\theta)$ can also be bounded by constants. Thus, there exists $m_{1}, m_{2} \in \mathcal{R}^{+}$ such that

$$
\begin{aligned}
m_{1}\|\boldsymbol{x}\|^{2} & \leq \boldsymbol{x}^{T} \mathbf{M}(\theta) \boldsymbol{x} \leq m_{2}\|\boldsymbol{x}\|^{2} \quad \text { and } \\
\frac{1}{m_{2}}\|\boldsymbol{x}\|^{2} & \leq \boldsymbol{x}^{T} \mathbf{M}^{-1}(\theta) \boldsymbol{x} \leq \frac{1}{m_{1}}\|\boldsymbol{x}\|^{2}, \quad \forall \boldsymbol{x} \in \mathcal{R}^{2}
\end{aligned}
$$

2. $\mathbf{C}(\theta, \dot{\theta})$ can be upper bounded as follows

$$
\|\mathbf{C}(\theta, \dot{\theta})\| \leq c_{1} \dot{\theta}^{2}, \quad c_{1} \in \mathcal{R}^{+} .
$$

Also, there exist a matrix $\mathbf{V}_{m}(\theta, \dot{\theta})$ such that $\mathbf{C}(\theta, \dot{\theta})=$ $\mathbf{V}_{m}(\theta, \dot{\theta}) \dot{\boldsymbol{q}}$ and

$\boldsymbol{x}^{T}\left(\frac{1}{2} \dot{\mathbf{M}}(\theta)-\mathbf{V}_{m}(\theta, \dot{\theta})\right) \boldsymbol{x}=0, \quad \forall \boldsymbol{x} \in \mathcal{R}^{2}$

The property in Eq. (6) is the usual skew symmetric property of the Coriolis/centripetal matrix of Lagrange dynamics (Lewis et al., 2004).

3. The damping matrix $\mathbf{B}(\theta)$ is symmetric and positive semi definite. Also, there exists positive definite matrices $\underline{\mathbf{B}}$ and $\overline{\mathbf{B}}$ such that

$$
0<\boldsymbol{x}^{T} \underline{\mathbf{B}} \boldsymbol{x} \leq \boldsymbol{x}^{T} \mathbf{B}(\theta) \boldsymbol{x} \leq \boldsymbol{x}^{T} \overline{\mathbf{B}} \boldsymbol{x}, \quad \forall \boldsymbol{x} \in \mathcal{R}^{2} .
$$

4. The stiffness vector $\boldsymbol{K}(\boldsymbol{q})$ is Lipschitz continuous, i.e. there exists a positive constant $k_{2}$ such that

$$
\left\|\boldsymbol{K}\left(\boldsymbol{q}_{1}\right)-\boldsymbol{K}\left(\boldsymbol{q}_{2}\right)\right\| \leq k_{2}\left\|\boldsymbol{q}_{1}-\boldsymbol{q}_{2}\right\| .
$$

5. The unique static equilibrium point $\boldsymbol{q}_{0}=$ $\left[\begin{array}{lll}y_{\mathrm{s}_{0}} & \theta_{0} & d_{0}\end{array}\right]^{T}$ of the undisturbed system is known and is given by

$$
\boldsymbol{K}\left(\boldsymbol{q}_{0}\right)-\boldsymbol{G}\left(\theta_{0}\right)+\boldsymbol{e}_{3,3} u_{0}=\mathbf{0} .
$$

\section{System analysis}

This section presents the finite-gain stability analysis of the system described in the previous section. The disturbance $\mathbf{d}_{r}$ in Eq. (2) is assumed to be unknown a priori but bounded in the sense that $\mathbf{d}_{r} \in \mathcal{L}_{2}$. As a result, robust optimal control is considered in which the gain of the system is optimized under worst excitations: Ball and Helton (1989); Helton and James (1999); Soravia (1996); van der Schaft (1996). The following definition describes the notion of stability used in the subsequent analyses.

Finite-Gain $\mathcal{L}$-Stable (van der Schaft, 1996) Consider the nonlinear system

$\dot{\boldsymbol{x}}=f(\boldsymbol{x}, \boldsymbol{w})$

$z=h(\boldsymbol{x})$

where $\boldsymbol{x} \in \mathcal{R}^{n}, \boldsymbol{w} \in \mathcal{R}^{p}, \boldsymbol{z} \in \mathcal{R}^{m}$ are the state, input, and output vectors, respectively. The system in Eq. (10), with the mapping $M_{H}: \mathcal{L}_{\mathrm{e}}^{p} \rightarrow \mathcal{L}_{\mathrm{e}}^{m}$, is said to be finite-gain $\mathcal{L}$-stable if there exist real constants $\gamma, \beta \geq 0$ such that

$\left\|M_{H}(\boldsymbol{w})\right\|_{\mathcal{L}} \leq \gamma\|\boldsymbol{w}\|_{\mathcal{L}}+\beta$

where $\|.\|_{\mathcal{L}}$ denotes the $\mathcal{L}$ norm of a signal, and $\mathcal{L}_{\mathrm{e}}^{n}$ is the extended $\mathcal{L}$ space defined as

$\mathcal{L}_{\mathrm{e}}^{n}=\left\{\chi \mid \chi_{\tau} \in \mathcal{L}^{n}, \forall \tau \in[0, \infty)\right\}$

where $\chi_{\tau}$ is a truncation of $\chi$ given as

$\chi_{\tau}(t)=\left\{\begin{array}{cc}\chi(t) & 0 \leq t \leq \tau \\ 0 & t>\tau .\end{array}\right.$.

For the purpose of this paper, the $\mathcal{L}_{2}$-space is considered, hence the finite-gain $\mathcal{L}$-stability condition in Eq. (11) is rewritten as (van der Schaft, 1996)

$\left\|M_{H}(\boldsymbol{w})\right\|_{2} \leq \gamma\|\boldsymbol{w}\|_{2}+\beta$,

where $\|.\|_{2}$ denotes the $\mathcal{L}_{2}$ norm of a signal given by

$\|\chi\|_{2}=\left(\int_{0}^{\infty} \chi^{T}(t) \chi(t) \mathrm{d} t\right)^{\frac{1}{2}}$

$\gamma^{*}=\inf \left\{\gamma \mid\left\|M_{H}(\boldsymbol{w})\right\|_{2} \leq \gamma\|\boldsymbol{w}\|_{2}+\beta\right\}$ is the gain of the system, and, in the case of linear quadratic problems, is the $H_{\infty}$ norm of the system. Given an attenuation level $\gamma>0$, and the corresponding system dynamics, the objective is to show that 
Eq. (14) is satisfied for some $\beta>0$. This solution is approached from the perspective of dissipative systems (Ball and Helton, 1989; van der Schaft, 1996). The following definition describes the concept of dissipativity with respect to the system in Eq. (10).

Dissipativity The dynamics system Eq. (10) is dissipative with respect to a given supply rate $s(\boldsymbol{w}, \boldsymbol{z}) \in \mathcal{R}$, if there exists an energy function $V(\boldsymbol{x}) \geq 0$ such that, for all $\boldsymbol{x}\left(t_{0}\right)=$ $\boldsymbol{x}_{0}$ and $t_{f} \geq t_{0}$,

$V\left(\boldsymbol{x}\left(t_{f}\right)\right) \leq V\left(\boldsymbol{x}\left(t_{0}\right)\right)+\int_{t_{0}}^{t_{f}} s(\boldsymbol{w}, \boldsymbol{z}) \mathrm{d} t, \quad \forall \boldsymbol{w} \in \mathcal{L}_{2}$.

If the supply rate is taken as

$s(\boldsymbol{w}, z)=\gamma^{2}\|\boldsymbol{w}\|^{2}-\|z\|^{2}$,

then the dissipation inequality in Eq. (16) implies finite-gain $\mathcal{L}$-stability (van der Schaft, 1996), and the system is said to be $\gamma$-dissipative. The dissipativity inequality is then written as

$\dot{V} \leq \gamma^{2}\|\boldsymbol{w}\|^{2}-\|z\|^{2}$.

\subsection{Performance objective}

As usual with suspension systems designs, the performance criterion is expressed in terms of the ride comfort, suspension deflection, and dynamic tire force. The performance vector

$\boldsymbol{z}=\left[\begin{array}{c}\omega_{1} y_{\mathrm{cba}} \\ \omega_{2} y_{\mathrm{sd}} \\ \omega_{3} y_{\mathrm{dtf}}\end{array}\right]$

characterizes the ride comfort, suspension deflection, and road holding performances, where $\omega_{1}, \omega_{2}$, and $\omega_{3}$ are the respective user specified performance weights for car body acceleration $y_{\mathrm{cba}}$, suspension deflection $y_{\mathrm{sd}}$, and dynamic tire force $y_{\mathrm{dtf}}$. The ride comfort is characterized by the car body acceleration $\ddot{y}_{\mathrm{s}}$ which is approximated using the following high gain observer (Khalil, 1996):

$$
\begin{aligned}
& \varepsilon \dot{\boldsymbol{\eta}}=\mathbf{A} \boldsymbol{\eta}+\mathbf{b} \dot{y}_{\mathrm{s}}, \quad \boldsymbol{\eta}(0)=\mathbf{0} \\
& y_{\mathrm{cba}}=\frac{1}{\varepsilon} \mathbf{c}^{T} \boldsymbol{\eta}
\end{aligned}
$$

where

$\mathbf{A}=\left[\begin{array}{ll}-1 & 1 \\ -1 & 0\end{array}\right], \quad \mathbf{b}=\left[\begin{array}{l}1 \\ 1\end{array}\right], \quad \mathbf{c}=\left[\begin{array}{l}0 \\ 1\end{array}\right]$

The $\mathcal{L}_{2}$-norm of the car body acceleration can be upper bounded as (Khalil, 1996)

$\left\|y_{\text {cbal }}\right\|_{2} \leq c_{1}\left\|\dot{y}_{\mathrm{s}}\right\|_{2} \leq c_{1}\|\dot{\boldsymbol{e}}\|_{2}$, where

$c_{1}=\frac{2 \lambda_{\max }^{2}(\mathbf{P})\|\mathbf{b}\|_{2}\|\mathbf{c}\|_{2}}{\lambda_{\min }(\mathbf{P})}$

and $\mathbf{P}$ is the solution of the Lyapunov equation $\mathbf{P A}+\mathbf{A}^{\mathrm{T}} \mathbf{P}+\mathbf{I}=$ 0 , which is obtained as

$\mathbf{P}=\frac{1}{\varepsilon}\left[\begin{array}{cc}1 & \frac{1}{2} \\ \frac{1}{2} & \frac{3}{2}\end{array}\right]$

The suspension deflection is given as

$$
\begin{aligned}
y_{\mathrm{sd}}(t) & =\sqrt{l_{\mathrm{s}}^{2}(0)-l_{\mathrm{s}}^{2}(t)} \\
& =\left\{d(0)^{2}-d(t)^{2}-2 H x(\sin \theta(0)-\sin \theta(t))\right. \\
& -2 x(d(0) \cos \theta(0)-d(t) \cos \theta(t))\}^{\frac{1}{2}} \\
& \leq\left[\begin{array}{lll}
0 & k_{41} & k_{42}
\end{array}\right]\left[\begin{array}{c}
\left|y_{0_{\mathrm{s}}}-y_{\mathrm{s}}\right| \\
\left|\theta-\theta_{0}\right| \\
\left|d-d_{0}\right|
\end{array}\right],
\end{aligned}
$$

Using the Cauchy-Schwarz inequality, $y_{\mathrm{sd}}(t)$ can be upper bounded as

$y_{\mathrm{sd}}(t) \leq k_{4}\|\boldsymbol{e}\|$,

where $k_{41}, k_{42}$, and $k_{4}$ are positive constants, and $k_{4} \geq \sqrt{k_{41}^{2}+k_{42}^{2}}$.

The dynamic tire force is characterized using the tire deflection and is given by

$$
\begin{aligned}
y_{\mathrm{dtf}}(t) & =y_{\mathrm{u}}(0)-y_{\mathrm{u}}(t) \\
& =y_{0_{\mathrm{s}}}-y_{\mathrm{s}}+l_{D}\left(\sin \theta_{0}-\sin \theta\right) \\
& \leq\left[\begin{array}{lll}
1 & k_{5} & 0
\end{array}\right]\left[\begin{array}{c}
\left|y_{0_{\mathrm{s}}}-y_{\mathrm{s}}\right| \\
\left|\theta-\theta_{0}\right| \\
\left|d-d_{0}\right|
\end{array}\right],
\end{aligned}
$$

where $k_{5}$ is a positive constant. Using the Cauchy-Schwarz inequality, $y_{\mathrm{dff}}(t)$ can be upper bounded as

$y_{\mathrm{dtf}} \leq \sqrt{1+k_{5}^{2}}\|\boldsymbol{e}\|=k_{6}\|\boldsymbol{e}\|$.

Finally, the $\mathcal{L}_{2}$-norm of the performance vector in Eq. (19) can be upper bounded as

$\|z\|_{2} \leq \phi_{1}\|\dot{\boldsymbol{e}}\|_{2}+\phi_{2}\|\boldsymbol{e}\|_{2}$

where

$$
\begin{aligned}
& \phi_{1}=\omega_{1} c_{1} \\
& \phi_{2}=\omega_{2} k_{4}+\omega_{3} k_{6} .
\end{aligned}
$$




\subsection{Constant stiffness case}

Now, consider the constant stiffness case in which the control mass is locked at a given position $d$. As a result, the overall stiffness is constant for the entire trajectory of the system. For this case, the dynamics in Eq. (2) reduces to

$\mathbf{M}_{1}(\theta) \ddot{\boldsymbol{q}}_{1}+\boldsymbol{C}_{1}(\theta, \dot{\theta})+\mathbf{B}_{1}(\theta) \dot{\boldsymbol{q}}_{1}-\boldsymbol{K}_{1}\left(\boldsymbol{q}_{1}\right)+\boldsymbol{G}_{1}(\theta)=\boldsymbol{w}$,

where

$$
\begin{array}{r}
\mathbf{M}_{1}=\mathbf{M}_{1: 2,1: 2}, \boldsymbol{C}_{1}=\boldsymbol{C}_{1: 2}, \\
\boldsymbol{K}_{1}=\boldsymbol{K}_{1: 2}, \mathbf{B}_{1}=\mathbf{B}_{1: 2,1: 2,}, \\
\boldsymbol{w}=\mathbf{W}_{d_{1}} \boldsymbol{d}_{r}, \mathbf{W}_{d_{1}}=\mathbf{W}_{d_{1: 2,1: 2}}
\end{array}
$$

Here, the corresponding dynamics of the control mass has been eliminated.

Let

$\boldsymbol{e}_{1}=\boldsymbol{q}_{1}-\boldsymbol{q}_{0_{1}}$

where

$\boldsymbol{q}_{0_{1}}=\left[\begin{array}{c}y_{\mathrm{s}_{0}} \\ \theta_{0}\end{array}\right]$

be the equilibrium value of the reduced state vector $\boldsymbol{q}_{1}$. After using the Mean Value Theorem, the closed-loop dynamics in Eq. (29) is expressed as

$\mathbf{M}_{1} \ddot{\boldsymbol{e}}_{1}+\mathbf{V}_{m_{1}} \dot{\boldsymbol{e}}_{1}+\mathbf{K}_{1} \boldsymbol{e}_{1}+\mathbf{B}_{1} \dot{\boldsymbol{e}}_{1}=\boldsymbol{w}$

where

$\mathbf{K}_{1}=-\left.\frac{\partial \boldsymbol{K}_{1}}{\partial \boldsymbol{q}_{1}}\right|_{\boldsymbol{q}_{1}=\zeta_{1}}+\left.\frac{\partial \boldsymbol{G}_{1}}{\partial \boldsymbol{q}_{1}}\right|_{\boldsymbol{q}_{1}=\zeta_{2}} \zeta_{1}, \zeta_{2}, \in \mathcal{L}_{\mathrm{s}}\left(\boldsymbol{q}_{0_{1}}, \boldsymbol{q}_{1}\right)$.

Lemma 1: The matrix

$\mathbf{P}=\left[\begin{array}{cc}\mathbf{I} & m_{1} \mathbf{I} \\ m_{1} \mathbf{I} & \mathbf{M}_{1}\end{array}\right]$

is positive definite, where $m_{1}^{2}<\lambda_{\min }\left\{\mathbf{M}_{1}\right\}$.

Proof. Let $\lambda$ be an eigenvalue of $\mathbf{P}$. It follows that $\lambda \in \mathcal{R}$, since $\mathbf{P}$ is symmetric. The characteristic polynomial of $\mathbf{P}$ is given by

$$
\begin{aligned}
p(\lambda) & =\operatorname{det}\{\lambda \mathbf{I}-\mathbf{P}\} \\
& =\operatorname{det}\left\{(\lambda-1)(\lambda \mathbf{I}-\mathbf{M})-m_{1}^{2} \mathbf{I}\right\}
\end{aligned}
$$

Now, $\lambda=1 \Rightarrow p(\lambda)=m_{1}^{4}$, which implies that $\lambda=1$ is NOT an eigenvalue of $\mathbf{P}$. Suppose without loss of generality that $\lambda \neq 1$, then

$p(\lambda)=(\lambda-1)^{2} \operatorname{det}\left\{\frac{\lambda^{2}-\lambda-m_{1}^{2}}{\lambda-1} \mathbf{I}-\mathbf{M}\right\}$.
Thus there exists an eigenvalue $\lambda_{m}$ of $\mathbf{M}$ such that

$\frac{\lambda^{2}-\lambda-m_{1}^{2}}{\lambda-1}=\lambda_{m}$

which implies that

$\lambda=\frac{1}{2}\left(1+\lambda_{m} \pm \sqrt{\left(1+\lambda_{m}\right)^{2}-4\left(\lambda_{m}-m_{1}^{2}\right)}\right)$,

from which it follows that $\lambda>0$. Since $\mathbf{P}$ is symmetric, the conclusion follows.

Remark It follows from Rayleigh-Ritz Inequality that

$p_{1}\|\chi\|^{2} \leq \chi^{T} \mathbf{P} \chi \leq p_{2}\|\chi\|^{2}$,

where $p_{1}=\lambda_{\min }\{\mathbf{P}\}$, and $p_{2}=\lambda_{\max }\{\mathbf{P}\}$.

Theorem 1. If the matrix

$\mathbf{H}_{1}=\frac{1}{2}\left[\begin{array}{cc}-\hat{\mathbf{K}}_{1}-\hat{\mathbf{K}}_{1}^{T} & -\mathbf{K}_{1}^{T}-m_{1} \mathbf{M}_{1}^{-1} \mathbf{B}_{1} \\ -\mathbf{K}_{1}-m_{1}\left(\mathbf{M}_{1}^{-1} \mathbf{B}_{1}\right)^{T} & -2 \hat{\mathbf{B}}_{1}\end{array}\right]$,

where

$\hat{\mathbf{K}}_{1}=m_{1} \mathbf{M}_{1}^{-1} \mathbf{K}_{1}-\frac{c_{1}\|\dot{\boldsymbol{e}}\|}{2} \mathbf{I}$

$\hat{\mathbf{B}}_{1}=\mathbf{B}_{1}-\left(m_{1}+\frac{c_{1}\|\dot{\boldsymbol{e}}\|}{2}\right) \mathbf{I}$,

is negative definite along the entire trajectory of the closedloop error system in Eq. (32), then the $\mathcal{L}_{2}$-norm of the performance vector in Eq. (19) can be upper bounded as

$\|\boldsymbol{z}\|_{2} \leq \gamma_{1}\|\boldsymbol{w}\|_{2}+\beta_{1}$,

where

$\gamma_{1}=\frac{\phi \sigma p_{2}}{p_{1} h_{1}}$,

$\beta_{1}=\frac{\sqrt{2} \phi p_{2}}{\sqrt{p_{1} h_{1}}}$,

and

$$
\begin{aligned}
\phi & =\max \left\{\phi_{1}, \phi_{2}\right\} \\
\sigma & =\sigma_{\max }\left\{\left[\begin{array}{c}
m_{1} \mathbf{M}_{1}^{-1} \\
\mathbf{I}
\end{array}\right]\right\} \\
h_{1} & =\left|\lambda_{\min }\left\{\mathbf{H}_{1}\right\}\right| .
\end{aligned}
$$

Proof. Consider the energy function

$V\left(\boldsymbol{e}_{1}, \dot{\boldsymbol{e}}_{1}\right)=\frac{1}{2} \chi_{1}^{T} P \chi_{1}$,

where

$\chi_{1}=\left[\begin{array}{c}e_{1} \\ \dot{e}_{1}\end{array}\right]$. 
Taking time derivative of Eq. (49) and using the skew symmetric property in Eq. (6) yields

$$
\begin{aligned}
\dot{V} & =-\dot{\boldsymbol{e}}_{1}^{T}\left(\mathbf{B}_{1}-m_{1} \mathbf{I}\right) \dot{\boldsymbol{e}}_{1}-\boldsymbol{e}_{1}^{T} \mathbf{K}_{1} \boldsymbol{e}_{1}+\dot{\boldsymbol{e}}_{1}^{T} \boldsymbol{w}+m_{1} \boldsymbol{e}_{1}^{T} \mathbf{M}_{1}^{-1} \boldsymbol{w} \\
& -m_{1} \boldsymbol{e}_{1}^{T} \mathbf{M}_{1}^{-1} \mathbf{V}_{m} \dot{\boldsymbol{e}}_{1}-m_{1} \boldsymbol{e}_{1}^{T} \mathbf{M}_{1}^{-1} B_{1} \dot{\boldsymbol{e}}_{1}-m_{1} \boldsymbol{e}_{1}^{T} \mathbf{M}_{1}^{-1} \mathbf{K}_{1} \boldsymbol{e}_{1} .
\end{aligned}
$$

Using the property in Eq. (5) yields

$$
\dot{V} \leq \chi_{1}^{T} \mathbf{H}_{1} \chi_{1}+\chi_{1}^{T}\left[\begin{array}{c}
m_{1} \mathbf{M}_{1}^{-1} \\
\mathbf{I}
\end{array}\right] \boldsymbol{w}
$$

which after using the negative definiteness of $H_{1}$ yields

$\dot{V} \leq-h_{1}\left\|\chi_{1}\right\|^{2}+\sigma\left\|\chi_{1}\right\|\|\boldsymbol{w}\|$.

Take $W(t)=\sqrt{V\left(\chi_{1}\right)}$. When $V\left(\chi_{1}\right) \neq 0, \dot{W}=\dot{V} /(2 \sqrt{V})$ yields

$\dot{W} \leq-\frac{h_{1}}{2 p_{2}} W+\frac{\sigma}{2 \sqrt{p_{1}}}\|\boldsymbol{w}\|$.

When $V\left(\chi_{1}\right)=0$, it can be verified (Khalil, 1996) that

$D^{+} W \leq \frac{\sigma}{2 \sqrt{p_{1}}}\|\boldsymbol{w}\|$,

where $D^{+}$denotes the upper right hand differentiation operator. Hence

$D^{+} W \leq-\frac{h_{1}}{2 p_{2}} W+\frac{\sigma}{2 \sqrt{p_{1}}}\|\boldsymbol{w}\|$

for all values of $V\left(\chi_{1}\right)$. Next using comparison (Lemma 3.4, Khalil, 1996) yields

$$
\begin{aligned}
W(t) \leq W(0) & \exp \left(-\frac{h_{1} t}{2 p_{2}}\right) \\
& +\frac{\sigma}{2 \sqrt{p_{1}}} \int_{0}^{t}\|\boldsymbol{w}\| \exp \left(-\frac{h_{1}(t-\tau)}{2 p_{2}}\right) \mathrm{d} \tau,
\end{aligned}
$$

which implies that

$$
\begin{aligned}
\left\|\chi_{1}(t)\right\| \leq \sqrt{\frac{p_{2}}{p_{1}}}\left\|\chi_{1}(0)\right\| \exp \left(-\frac{h_{1} t}{2 p_{2}}\right) \\
+\frac{\sigma}{2 p_{1}} \int_{0}^{t}\|\boldsymbol{w}\| \exp \left(-\frac{h_{1}(t-\tau)}{2 p_{2}}\right) \mathrm{d} \tau .
\end{aligned}
$$

Thus

$$
\left\|\chi_{1}(t)\right\|_{2} \leq \frac{\sigma p_{2}}{p_{1} h_{1}}\|\boldsymbol{w}\|_{2}+\frac{\sqrt{2} p_{2}}{\sqrt{p_{1} h_{1}}}\left\|\chi_{1}(0)\right\| .
$$

Lastly, after using the inequality in Eq. (28), the $\mathcal{L}_{2}$-norm of the performance vector can be upper bounded as

$\|z\|_{2} \leq \frac{\phi \sigma p_{2}}{p_{1} h_{1}}\|\boldsymbol{w}\|_{2}+\frac{\sqrt{2} \phi p_{2}}{\sqrt{p_{1} h_{1}}}\left\|\chi_{1}(0)\right\|$.
Remark The $\mathcal{L}_{2}$-gain of the system decreases with increasing $h_{1}$. This means that the more the negative definiteness of $H_{1}$, the more the disturbance rejection achievable by the system.

The following theorem gives the bounds on achievable $\gamma$.

Theorem 2. Given an attenuation level $\gamma$, and provided that the performance weights are selected to satisfy the sufficient condition

$\phi=\max \left\{\phi_{1}, \phi_{2}\right\}<\sqrt{h_{1}}$,

then the closed loop error system in Eq. (32) is $\gamma$-dissipative with respect to the supply rate

$s(\boldsymbol{w}, \boldsymbol{z})=\gamma^{2}\|\boldsymbol{w}\|^{2}-\|z\|^{2}$

if

$\gamma \geq \frac{0.5 \sigma}{\sqrt{h_{1}-\phi^{2}}}$.

Proof. Consider the energy storage function in (49). Taking first time-derivate, and adding and subtracting the supply rate yields

$$
\begin{aligned}
\dot{V} & \leq \chi^{T} \mathbf{H}_{1} \chi+\chi^{T} L \boldsymbol{w} \\
& \leq \gamma^{2}\|\boldsymbol{w}\|^{2}-\|\boldsymbol{z}\|^{2}+\chi^{T} \mathbf{H}_{1} \chi \\
& \quad-\gamma^{2}\left\|\boldsymbol{w}-\frac{L^{T} \chi}{2 \gamma^{2}}\right\|^{2}+\frac{1}{4 \gamma^{2}} \chi^{T} L L^{T} \chi+\phi^{2}\|\chi\|^{2} \\
& \leq \gamma^{2}\|\boldsymbol{w}\|^{2}-\|z\|^{2}+\chi^{T}\left(\mathbf{H}_{1}+\left(\phi^{2}+\frac{\sigma^{2}}{4 \gamma^{2}}\right) I\right) \chi \\
& \leq \gamma^{2}\|\boldsymbol{w}\|^{2}-\|z\|^{2}-\left(h_{1}-\phi^{2}-\frac{\sigma^{2}}{4 \gamma^{2}}\right)\|\chi\|^{2}
\end{aligned}
$$

After using the inequality in Eq. (62)

$\dot{V} \leq \gamma^{2}\|\boldsymbol{w}\|^{2}-\|z\|^{2}$,

which implies that the closed loop error system in Eq. (32) is $\gamma$-dissipative.

Remark The inequality in Eq. (62) shows that the level of performance achievable is limited by the amount of damping and stiffness available in the system. It will be shown in subsequent sections that this limit can be pushed further by using a variable stiffness architecture. The lower bound in Eq. (62) is termed "best-case-gain". It defines the smallest gain achievable by the system.

The stiffness and damping matrices $\mathbf{K}_{1}$, and $\mathbf{B}_{1}$ contain bounded functions of state and uncertain dynamic parameters which range between bounded values. Thus the bestcase gain of the system with constant stiffness can be lower bounded as

$\underline{\gamma}_{1} \geq \frac{0.5 \sigma}{\sqrt{h_{1}^{*}-\phi^{2}}}$. 
where $h_{1}^{*}$ is the smallest positive number larger than the smallest singular value of $\mathbf{H}_{1}$, and $\underline{\gamma}_{1}$ is termed the "robust best-case gain".

\subsection{Passive variable stiffness case}

Here, the control mass is allowed to move under the influence of a restoring spring and damper forces. There is no external force generator added to the system. As a result, the system response is purely passive. Let $k_{u}$ and $b_{u}$ be the spring constant and damping coefficient of the restoring spring and damper respectively. The control force $u$ is then given by

$u=-b_{u} \dot{d}-k_{u}\left(d-l_{0_{d}}\right)$,

and the resulting dynamics of the control mass is given by

$$
\begin{array}{r}
m_{d} \ddot{d}+b_{u} \dot{d}+k_{u}\left(d-l_{0_{d}}\right)+k_{\mathrm{s}}\left(\rho_{\mathrm{s}}-1\right)(d-x \cos \theta) \\
+\frac{b_{\mathrm{s}}}{2} g_{d \theta} \dot{\theta}+b_{\mathrm{s}} g_{d} \dot{d}=0,
\end{array}
$$

and the static equilibrium equation for the control mass is given by

$k_{u}\left(d_{0}-l_{0_{d}}\right)+k_{\mathrm{s}}\left(\rho_{s_{0}}-1\right)\left(d_{0}-x \cos \theta_{0}\right)=0$,

where $d_{0}$ is the equilibrium position of the control mass, and $l_{0_{d}}$ is the free length of the restoring spring. Let

$e_{d}=d-d_{0}$

be the displacement of the control mass from its equilibrium position. Substituting Eq. (69) into Eq. (67) and using the Mean Value Theorem yields

$m_{d} \ddot{e}_{d}+\boldsymbol{B}_{d}^{T} \dot{\boldsymbol{e}}+\boldsymbol{K}_{d}^{T} \boldsymbol{e}=0$,

where

$$
\begin{aligned}
\boldsymbol{e} & =\left[\begin{array}{c}
\mathbf{e}_{1} \\
e_{d}
\end{array}\right], \\
\boldsymbol{B}_{d} & =\left[\begin{array}{c}
0 \\
\frac{b_{\mathrm{s}}}{2} g_{d \theta} \\
b_{\mathrm{s}} g_{d}+b_{u}
\end{array}\right],
\end{aligned}
$$

$\boldsymbol{K}_{d}=\left[\begin{array}{c}0 \\ \left.k_{\mathrm{s}} \frac{\partial\left(\rho_{\mathrm{s}}-1\right)(d-x \cos \theta)}{\partial \theta}\right|_{\theta \in \mathcal{L}_{\mathrm{s}}\left(\theta_{0}, \theta\right)} \\ k_{u}+\left.k_{\mathrm{s}} \frac{\partial\left(\rho_{\mathrm{s}}-1\right)(d-x \cos \theta}{\partial d}\right|_{d \in \mathcal{L}_{\mathrm{s}}\left(d_{0}, d\right)}\end{array}\right]$.

Now, consider the energy function

$V_{2}(\boldsymbol{e}, \dot{\boldsymbol{e}})=\cdot \chi_{2}^{T} \mathbf{P}_{2} \chi_{2}$,

where,

$\chi_{2}=\left[\begin{array}{c}\boldsymbol{e} \\ \dot{e}\end{array}\right]$, and

$\mathbf{P}_{2}=\left[\begin{array}{cc}\mathbf{I} & m \mathbf{I} \\ m \mathbf{I} & \mathbf{M}\end{array}\right]$

is positive definite, with $m^{2}<\lambda_{\min }\{\mathbf{M}\}$. Taking the first time derivative of Eq. (74), and following a similar procedure as in the constant stiffness case in the previous section yields

$\dot{V}_{2} \leq \gamma^{2}\|\boldsymbol{w}\|^{2}-\|z\|^{2}+\chi_{2}^{T} \mathbf{H}_{2} \chi_{2}$,

where

$$
\begin{aligned}
\mathbf{H}_{2} & =\frac{1}{2}\left[\begin{array}{cc}
-\hat{\mathbf{K}}-\hat{\mathbf{K}}^{T} & -\mathbf{K}^{T}-m \mathbf{M}^{-1} \mathbf{B} \\
-\mathbf{K}-m\left(\mathbf{M}^{-1} \mathbf{B}\right)^{T} & -2 \hat{\mathbf{B}}
\end{array}\right], \\
\hat{\mathbf{K}} & =m \mathbf{M}^{-1} \mathbf{K}-\frac{c_{1} \| \dot{\boldsymbol{e} \|}}{2} \mathbf{I}, \\
\hat{\mathbf{B}} & =\mathbf{B}-\left(m_{1}+\frac{c_{1}\|\dot{\mathbf{e}}\|}{2}\right) \mathbf{I},
\end{aligned}
$$

and

$$
\mathbf{K}=-\left.\frac{\partial \boldsymbol{K}}{\partial \boldsymbol{q}}\right|_{\boldsymbol{q}=\zeta_{1}}+\left.\frac{\partial \boldsymbol{G}}{\partial \boldsymbol{q}}\right|_{\boldsymbol{q}=\zeta_{2}}, \boldsymbol{\zeta}_{1}, \zeta_{2}, \in \mathcal{L}_{\mathrm{s}}\left(\boldsymbol{q}_{0}, \boldsymbol{q}\right) .
$$

Now, the robust best-case gain of the system with a passive variable stiffness is given by

$\underline{\gamma}_{2} \geq \frac{0.5 \sigma}{\sqrt{h_{2}^{*}-\phi^{2}}}$.

where $h_{2}^{*}$ is the smallest positive number larger than smallest singular value of $\mathbf{H}_{2}$. Here, the spring constant $k_{u}$, and the damping coefficient $b_{u}$ of the control mass restoring springdamper system can be chosen such that $\underline{\gamma}_{2}<\underline{\gamma}_{1}$. Thus, a better performance can be achieved just by letting the stiffness vary naturally using a spring-damper system. This claim is supported subsequently by experimental and simulation results. This is a very appealing result due to its practicability. No additional electronically controlled or force generating device is required, only mechanical elements like the spring and damper are used.

\section{Experiment}

The experimental setup is shown in Fig. 4. It is a quarter car test rig scaled down to a ratio of 1:10 compared to an average passenger car in 2004 (NHTSA, 2004). The quarter car body is allowed to translate up-and-down along a rigid frame. This was made possible through the use of two pairs of linear motion ball-bearing carriages, with each pair on separate parallel guide rails. The guide rails are fixed to the rigid frame and the carriages are attached to the quarter car frame. The quarter car frame is made of 80/20 aluminium framing and then loaded with a solid steel cylinder weighing approximately $80 \mathrm{lbs}$. The horizontal and vertical struts are 


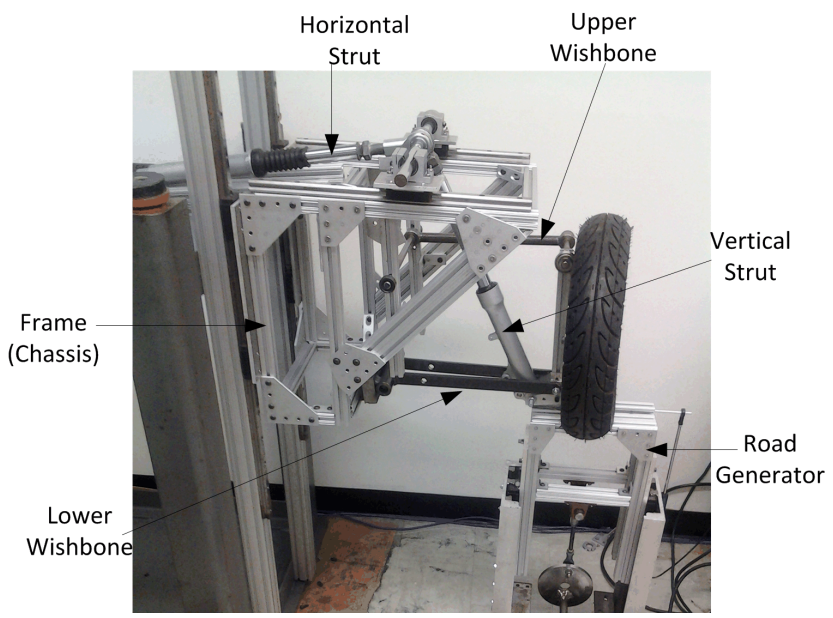

Figure 4. Quarter Car Experimental Setup.

the 2011 Honda PCX scooter front suspensions. The road generator is a simple slider-crank mechanism actuated by Smartmotor ${ }^{\circledR}$ SM3440D geared down to a ratio of $49: 1$ using $\mathrm{CMI}^{\circledR}$ gear head P/N 34EP049. Three accelerometers are attached, one each to the quarter car frame, the wheel hub, and the road generator. Data acquisition was done using the MATLAB data acquisition toolbox via NI USB-6251. Experiments were performed for the passive case, where the horizontal strut is just a passive spring-damper system, and also for the fixed stiffness case, where the top of the vertical strut is locked in a fixed position. This position is the equilibrium position of the passive case when the system is not excited.

Two tests were carried out; sinusoidal, and drop test. For the sinusoidal test, the road generator is actuated by a constant torque from the DC motor. As a result, the quarter car frame moves up and down in a sinusoidal fashion. To facilitate a good comparison of the observations, the "approximate gain" of the system defined as

$\gamma^{2}=\frac{\int_{0}^{T} z(t)^{2} \mathrm{~d} t}{\int_{0}^{T} r(t)^{2} \mathrm{~d} t}$,

where $z(t)$ is the signal of interest, and $r(t)$ is the road acceleration signal, is numerically computed. The signals of interest are the frame acceleration and tire deflection acceleration signals. The experimental procedure was repeated multiple times in order to verify the repeatability of the experiment. Figures 5 and 6 show the box plots of the approximate gain distributions for the fixed stiffness and passive variable stiffness cases. It is seen that the worst and best case gains for the fixed stiffness are higher than those of the passive variable stiffness case, thereby confirming the analytical result obtained earlier that the variable stiffness achieves better dissipation.

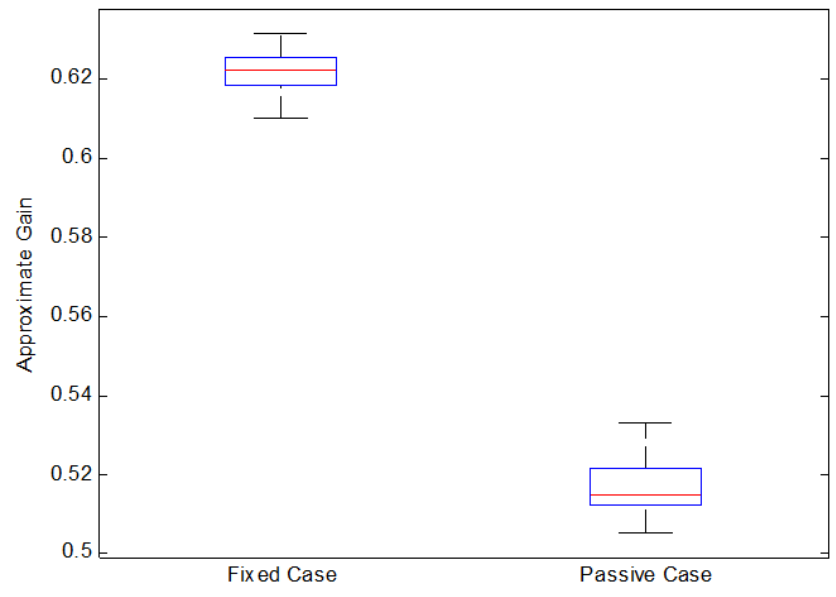

Figure 5. Box Plot: Car Body Acceleration.

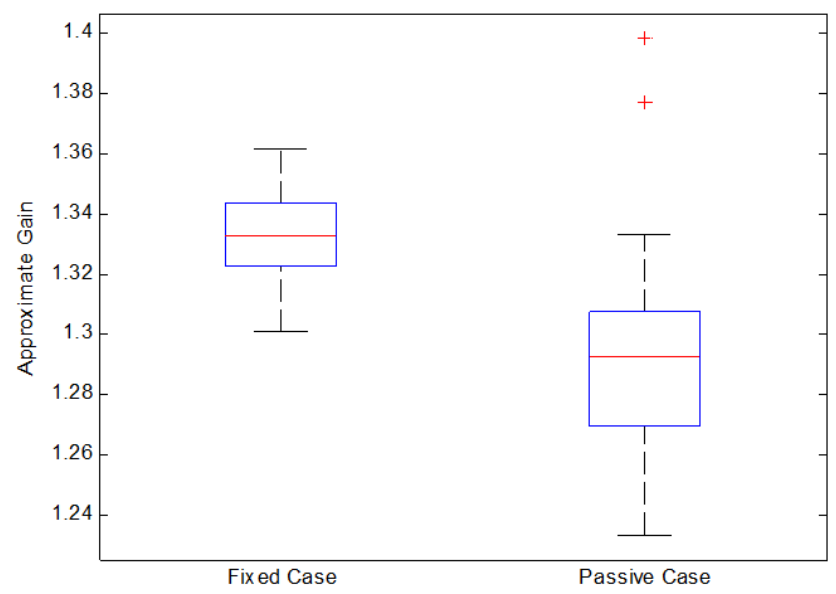

Figure 6. Box Plot: Tire Deflection Acceleration.

For the drop test, the suspension system was dropped to the ground ${ }^{1}$ from a fixed height ( 6 inches from the equilibrium position and the wheel was not in contact with the ground). The resulting quarter car body acceleration and tire deflection accelerations were recorded. This test examines the response of the system to initial conditions. Figures 7 and 8 shows the car body acceleration responses and tire deflection acceleration responses for the fixed and variable stiffness cases.

Table 1 shows the approximate gains for the sinusoidal and the rms values of the drop test. The approximate gains of the sinusoidal test given in the table are the mean values of the multiple experiments.

\section{Simulation}

In order to study the behavior of the quarter car system at full scale as well as responses like suspension deflection, which

\footnotetext{
${ }^{1}$ Here the ground is non accelerating as against the sinusoidal test where the ground simulates the road signal.
} 


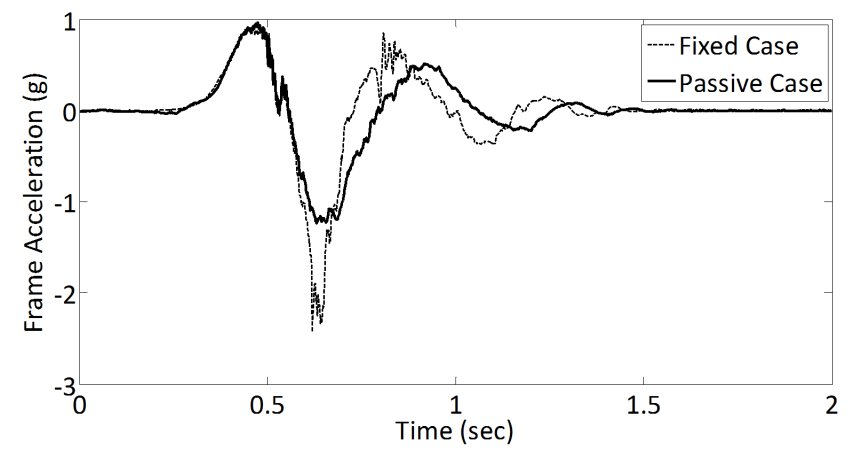

Figure 7. Drop Test: Car Body Acceleration.

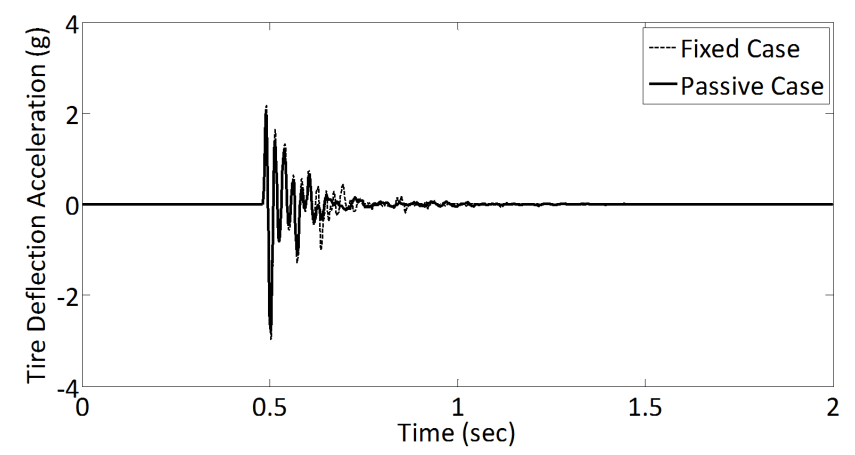

Figure 8. Drop Test: Tire Deflection Acceleration.

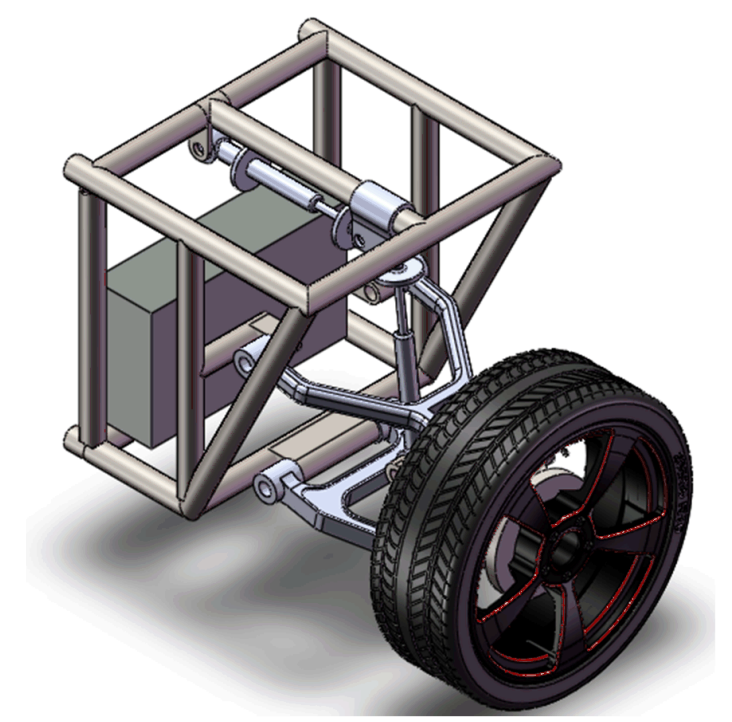

Figure 9. Solidworks Quarter Car Model.

were difficult to measure experimentally, and excitation scenarios that are difficult to implement experimentally, realistic simulations were carried out using MATLAB Simmechanics Second Generation. First, the system was modeled in Solidworks as shown in Fig. 9. Next, the Simmehanics model was developed. The mass/inertia properties used are the ones generated from the Solidworks model. The vertical strut and tire
Table 1. RMS/Approximate gain values of experimental results. CBA: Car Body Acceleration. TDA: Tire Deflection Acceleration.

\begin{tabular}{cccc}
\hline \multirow{2}{*}{ Drop (RMS) } & & Fixed & Passive \\
\cline { 2 - 4 } & CBA (g) & 0.4543 & 0.3710 \\
\cline { 2 - 4 } & TDA (g) & 0.2746 & 0.2396 \\
\hline \multirow{2}{*}{ Sinusoidal (Gain) } & CBA & 0.6220 & 0.5170 \\
\cline { 2 - 4 } & TDA & 1.3316 & 1.2944 \\
\hline
\end{tabular}

Table 2. Dynamic parameter values.

\begin{tabular}{cl}
\hline Parameter & Value \\
\hline$m_{\mathrm{s}}$ & $315 \mathrm{~kg}$ \\
$m_{\mathrm{u}}$ & $37.5 \mathrm{~kg}$ \\
$b_{\mathrm{s}}$ & $1500 \mathrm{~N} \mathrm{~m}^{-1} \mathrm{~s}^{-1}$ \\
$k_{\mathrm{s}}$ & $29500 \mathrm{~N} \mathrm{~m}^{-1}$ \\
$k_{\mathrm{t}}$ & $210000 \mathrm{~N} \mathrm{~m}^{-1}$ \\
\hline
\end{tabular}

damping and stiffness used are the ones given in the "Renault Mégane Coupé" model (Zin et al., 2004). The values are given in Table 2.

\subsection{Time domain simulation}

In the time domain simulation, the vehicle traveling at a steady horizontal speed of $40 \mathrm{mph}$ is subjected to a road bump of height $8 \mathrm{~cm}$. The Car Body Acceleration, Suspension Deflection, and Tire Deflection responses are compared between the constant stiffness and the passive variable stiffness cases. For the constant stiffness case, the control mass was locked at three different locations $(d=40 \mathrm{~cm}$, $d=45.56 \mathrm{~cm}$ and $d=50 \mathrm{~cm}$ ). The value $d=45.56 \mathrm{~cm}$ is the equilibrium position of the control mass. Next, a simulation is performed for the passive case. The results are reported in Figs. 10, 11 and 12 which are the the car body acceleration, suspension deflection, and tire deflection responses, respectively. Figure 13 shows the position history of the control mass for the passive variable stiffness case.

\subsection{Frequency domain simulation}

For the frequency domain simulation, an approximate frequency response from the road disturbance input to the performance vector given in Eq. (19), is computed using the notion of variance gain (Schoukens et al., 2001; Stack and Doyle, 1995). The approximate variance gain is given by 


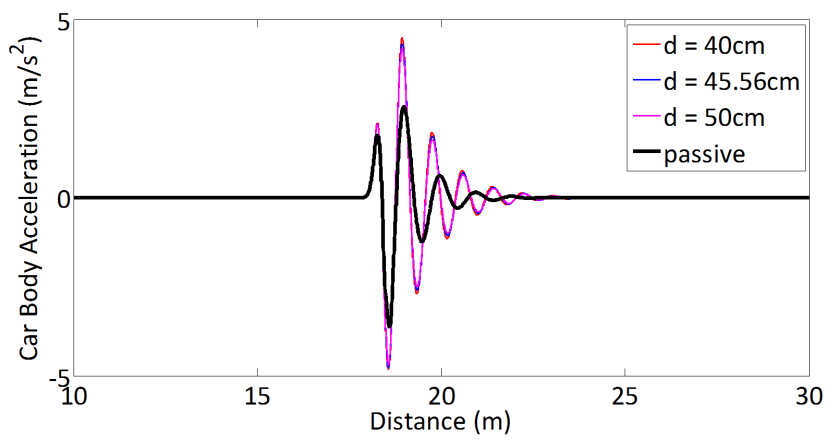

Figure 10. Time Domain Simulation: Car Body Acceleration.

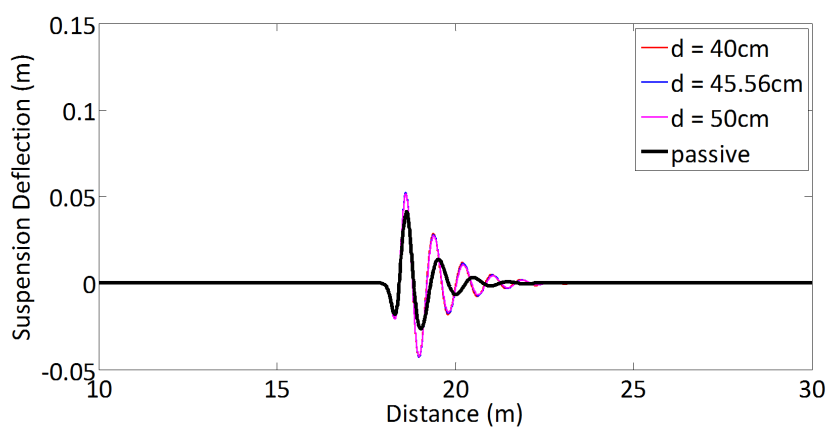

Figure 11. Time Domain Simulation: Suspension Deflection.

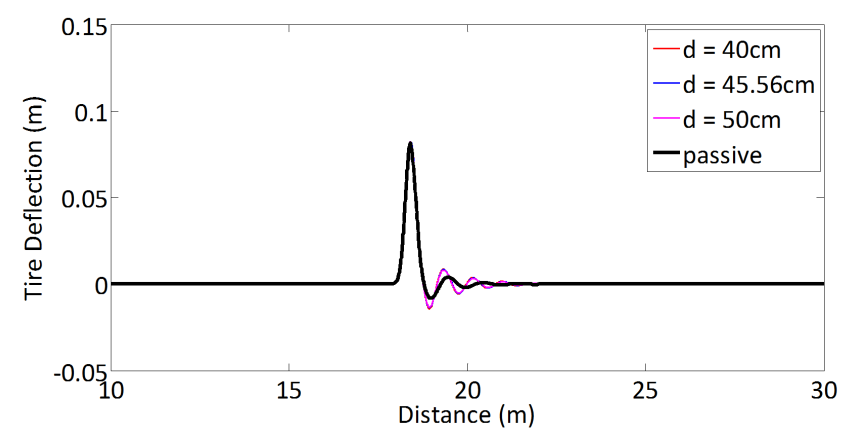

Figure 12. Time Domain Simulation: Tire Deflection.

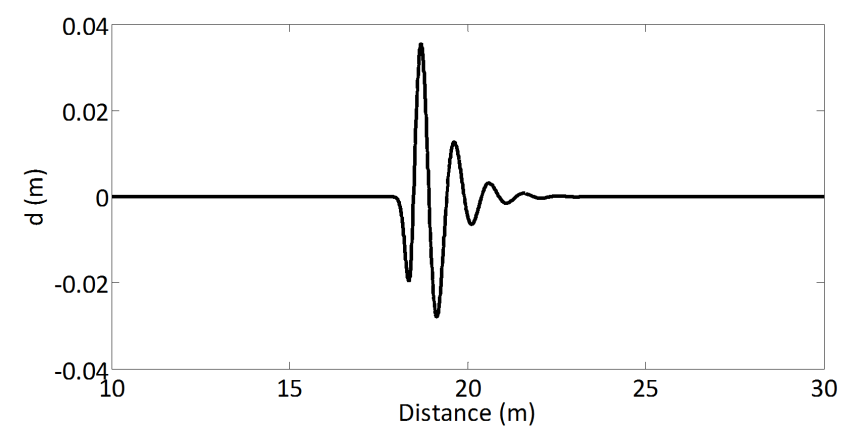

Figure 13. Time Domain Simulation: Control Mass Position.

$$
G(j \omega)=\sqrt{\frac{\int_{0}^{2 \pi N / \omega} z^{2} \mathrm{~d} t}{\int_{0}^{2 \pi N / \omega} A^{2} \sin ^{2}(\omega t) \mathrm{d} t}},
$$

where $z$ denotes the performance measure of interest which is taken to be car body acceleration, suspension deflection, and tire deflection. The closed loop system is excited by the sinusoid $r=A \sin (\omega t), t \in[0,2 \pi N / \omega]$, where $N$ is an integer big enough to ensure that the system reaches a steady state. The corresponding output signals were recorded and the approximate variance gains were computed using Eq. (84). Figures 14,15 , and 16 show the variance gain plots for the car body acceleration, suspension deflection, and tire deflection respectively. The figures show that the variable stiffness suspension achieves better vibration isolation in the human sensitive frequency range (4-8 Hz) (ISO 2631-1, 1997), and better handling beyond the tire hop frequency $(>59 \mathrm{~Hz})$ (Fialho and Balas, 2002).

\section{Conclusion}

The design, analysis, and experimentation of the passive case of a new variable stiffness suspension system is presented. Using a detailed $\mathcal{L}_{2}$-gain analysis based on the concept of energy dissipation, it is shown that inclusion of a variable stiffness mechanism in the suspension design yields an improvement in the performance of the traditional system in terms of ride comfort, suspension deflection, and road holding. The analysis claims are supported by both experimental and simulation results. In the future, work will be done on the semi-active case, where the passive spring/damper system will be replaced with a semi-active element like the MR damper. Also, the active case will be examined, where the horizontal strut will be replaced with a force generator such as hydraulic or pneumatic actuators. Moreover, the effect of nonlinear passive elements in the horizontal strut will be considered, and possibly considering nonlinear paths for the control mass as well. The effect of variable stiffness on roll and pitch dynamics will also be examined using a half-car model. 


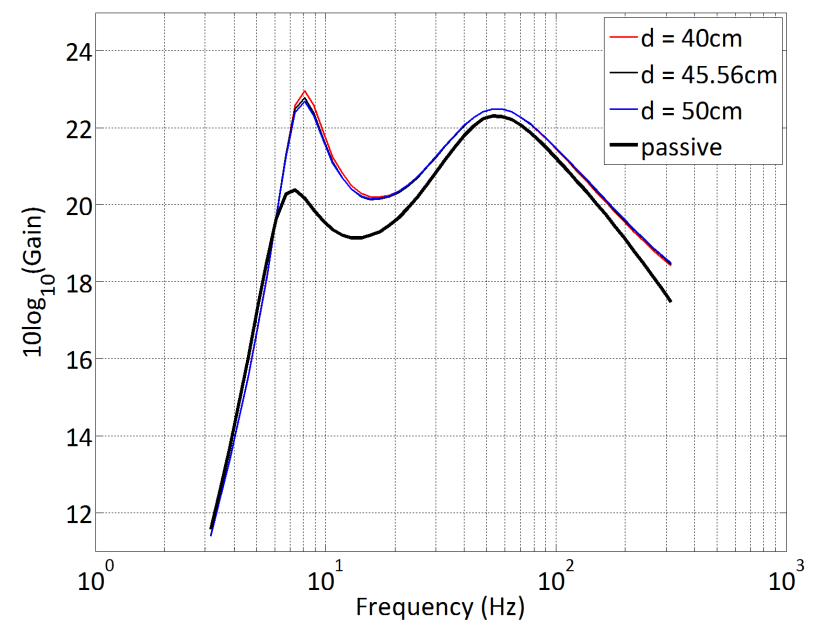

Figure 14. Frequency Domain Simulation: Car Body Acceleration.

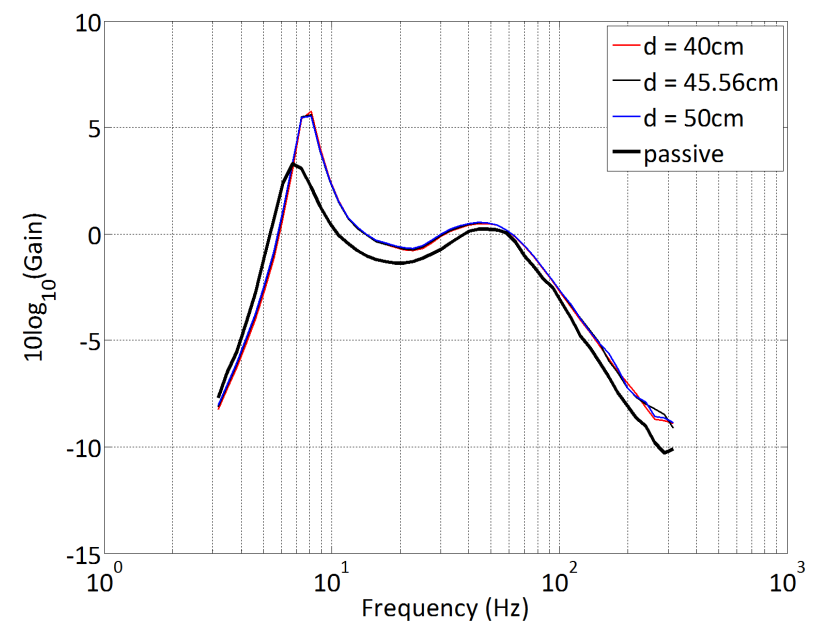

Figure 15. Frequency Domain Simulation: Suspension Deflection

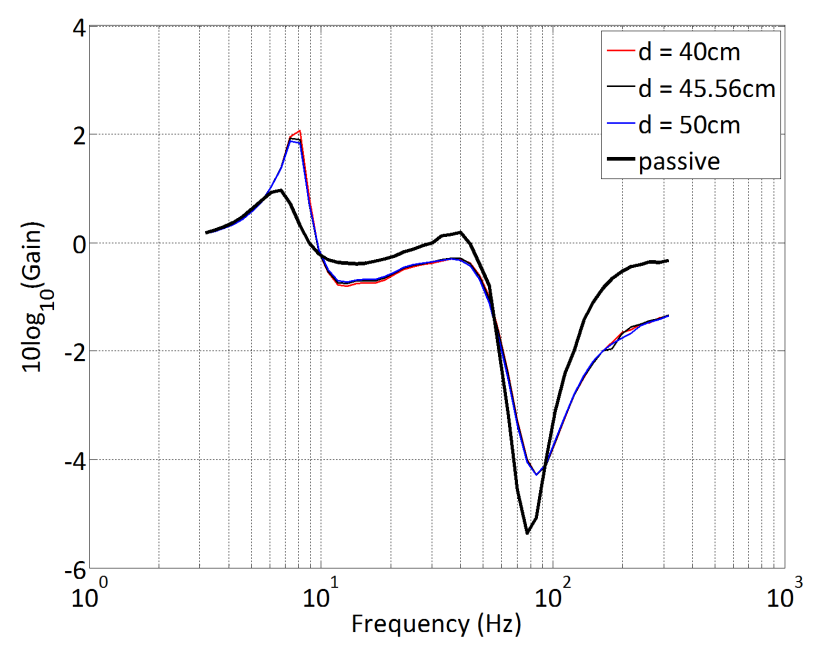

Figure 16. Frequency Domain Simulation: Tire Deflection.

\section{Nomenclature}

$\|\boldsymbol{v}\| \quad$ Euclidean norm of the vector $\boldsymbol{v}$

$y_{\mathrm{u}} \quad$ Vertical displacement of the unsprung mass

$y_{\mathrm{s}} \quad$ Vertical displacement of the sprung mass

$h_{u} \quad$ Half distance between points C and D

$l_{\mathrm{s}} \quad$ Vertical strut length

$l_{0_{\mathrm{s}}} \quad$ Natural length of vertical strut

$l_{D} \quad$ Length of the lower wishbone

$H \quad$ Height of the control mass from the pivot point of the lower wishbone

$x \quad$ Distance between points $\mathrm{O}$ and A along the lower wishbone

$k_{\mathrm{t}}, b_{\mathrm{t}} \quad$ Tire spring constant and damping coefficient

$k_{\mathrm{s}}, b_{\mathrm{s}} \quad$ Vertical Strut stiffness and damping coefficient

$k_{u}, b_{u} \quad$ Control(Horizontal) Strut stiffness and damping

$m_{\mathrm{s}}, m_{\mathrm{u}}, m_{d} \quad$ Sprung, unsprung and control masses

$I_{\mathrm{c}} \quad$ Moment of inertia of control arm.

$\lambda_{\min }\{\mathbf{A}\} \quad$ The minimum eigenvalue of the matrix $\mathbf{A}$

$\lambda_{\max }\{\mathbf{A}\} \quad$ The maximum eigenvalue of the matrix $\mathbf{A}$

$\sigma_{\min }\{\mathbf{A}\} \quad$ The minimum singular value of the matrix

A

$\sigma_{\max }\{\mathbf{A}\} \quad$ The maximum singular value of the matrix A

$\mathbf{A}_{i: j, k: l} \quad$ The sub-matrix of matrix $\mathbf{A}$ formed by rows $i$ to $j$ and columns $k$ to $l$

$\mathbf{A}_{i: j} \quad$ The sub-matrix of matrix $\mathbf{A}$ formed by rows $i$ to $j$ and all columns

$\operatorname{tr}\{\mathbf{A}\} \quad$ The trace of the matrix $\mathbf{A}$

$\operatorname{det}\{\mathbf{A}\} \quad$ The determinant of the matrix $\mathbf{A}$

$\mathcal{L}_{\mathrm{s}}\left(\boldsymbol{q}_{1}, \boldsymbol{q}_{2}\right) \quad$ The set of points that lie on the line segment joining the vectors $\boldsymbol{q}_{1}$ and $\boldsymbol{q}_{2}$

I Identity matrix

$\mathbf{e}_{i, n} \quad$ The $i$-th column of the identity matrix of dimension $n$

$\mathcal{R} \quad$ The set of real numbers

$\operatorname{Re}\{\alpha\} \quad$ The real part of the complex number $\alpha$

Edited by: A. Müller

Reviewed by: two anonymous referees 


\section{References}

Alkhatib, R., Jazar, G. N., and Golnaraghi, M. F.: Optimal design of Passive Linear Suspension Using Genetic Algorithm, J. Sound Vib., 275, 665-691, 2004.

Anubi, O. M., Crane, C., and Ridgeway, S.: Design and Analysis of a Variable Stiffness Mechanism, in: Proceedings IDETC/CIE2010. ASME 2010 International Design Engineering Technical Conferences \& Computers and Information in Engineering Conference, 2010.

Ashfak, A., Saheed, A., Abdul-Rasheed, K. K., and Jaleel, J. A.: Design, Fabrication and Evaluation of MR Damper, International Journal of Aerospace and Mechanical Engineering, 1, 27-33, 2011.

Ball, J. and Helton, J.: $H_{\infty}$ control for nonlinear plants: Connections with differential games, in: Proceedings of the 28th Conference on Decision and Control, Tampa, Florida, 956-962, 1989.

Butsuen, T.: The Design of Semi-active Suspensions for Automotive Vehicles, Ph.D. thesis, Massachussets Institute of Technology, 1989.

Do, A.-L., Sename, O., and Dugard, L.: An LPV Control Approach for Semi-active Suspension Control with Actuator Constraints, in: 2010 American Control Conference, 2010.

Evers, W.-J., Teehuis, A., van der Knaap, A., Besselink, I., and Nijmeijer, H.: The Electromechanical Low-Power Active Suspension: Modeling, Control, and Prototype Testing, J. Dyn. Syst.-T. ASME, 133, 041008-1-041008-9, 2011.

Fialho, I. and Balas, G. J.: Road Adaptive Active Suspension Design Using Linear Parameter-Varying Gain-Scheduling, IEEE T. Contr. Syst. T., 10, 43-54, 2002.

Helton, J. and James, M.: Extending $H_{\infty}$ Control to Nonlinear Systems: Control of Nonlinear Systems to Achieve Performance Objectives, Society for Industrial Mathematics, 1987.

ISO 2631-1, I.: International Organization of Standardization. Mechanical Vibration and Shock - Evaluation of Human Exposure to Whole Body Vibration. Part 1: General Requirement, Geneva, 1997.

Jerz, J.: Variable stiffness suspension system, uS Patent 3,559,976, 1971.

Karnopp, D.: Active damping in road vehicle suspension systems, Vehicle System Dynamics, 12, 291-316, 1983.

Karnopp, D. and Heess, G.: Electronically controllable vehicle suspensions, Vehicle Syst. Dyn., 20, 207-217, 1991.

Karnopp, D., Crosby, M., and Harwood, R.: Vibration control using semi-active force generators, J. Eng. Ind., 96, 619-626, 1974.

Khalil, H.: Nonlinear systems, Prentice hall New Jersey, 3rd Edn., 1996.

Kobori, T., Takahashi, M., Nasu, T., Niwa, N., and Ogasawara, K.: Seismic response controlled structure with active variable stiffness system, Earthq. Eng. Struct. D., 22, 925-941, 1993.

Lewis, F. L., Dawson, D. M., and Abdallah, C. T.: Robot Manipulator Control, Theory and Practice, Marcel Dekker, Inc., 2nd Edn., 2004.
Liu, Y., Matsuhisa, H., and Utsuno, H.: Semi-active vibration isolation system with variable stiffness and damping control, J. Sound Vib., 313, 16-28, doi:10.1016/j.jsv.2007.11.045, 2008.

NHTSA: New Passenger Car Fleet Average Characterisitcs, http: //www.nhtsa.gov/cars/rules/cafe/NewPassengerCarFleet.htm, 2004.

Schoukens, J., Pintelon, R., Rolain, Y., and Dobrowiecki, T.: Frequency Response Function Measurements in the Presence of Nonlinear Distortions, Automatica, 37, 939-946, 2001.

Soravia, P.: $H_{\infty}$ Control for Nonlinear Systems: Differential and Viscosity Solutions, SIAM Journal of Control and Optimization, 34, 1071-1097, 1996.

Stack, A. J. and Doyle, F. J.: A Measure for Control Relevant Nonlinearity, in: American Control Conferencel, Seattle, 2200-2204, 1995.

Sung, K., Han, Y., Lim, K., and Choi, S.: Discrete-time fuzzy sliding mode control for a vehicle suspension system featuring an electrorheological fluid damper, VTT Symp., 16, 798-808, 2007.

Tseng, H. E. and Hedrick, J. K.: Semi-Active Control Laws - Optimal and Sub-Optimal, Vehicle Syst. Dyn., 23, 545-569, 1994.

Valasek, M. and Kortum, W.: Nonlinear control of semi-active roadfriendly truck suspension, in: Proceedings AVEC 98, 275-280, Nagoya, 1998.

Valasek, M. and Kortum, W.: The Mechanical Systems Design Handbook; Modeling, Measurement and Control, chap. SemiActive Suspension Systems II, CRC Press LLC, 2001.

van der Knaap, A.: Design of a Low Power Anti-Roll/Pitch System for a Passenger Car, Ph.D. thesis, Delft University of Technology, 1989.

van der Knaap, A. C. M., Teerhuis, A. P., Tinsel, R. B. G., and Vershuren, R. M. A. F.: Active Suspension Assembly for a Vehicle, International Patent No. 2008/049845, 2008.

van der Schaft, A. J.: $\mathcal{L}_{2}$-Gain and Passivity Techniques in Nonlinear Control, Springer, Berlin, 1996.

Venhovens, P. J. T. and van der Knaap, A. C. M.: Delft Active Suspension (DAS), Background Theory and Physical Realization, Smart Vehicles, 139-165, 1995.

Williams, R. A.: Automotive active suspensions. Part 1: basic principles, in: Proceedings of IMechE, 211, 415-426, 1997.

Williams, R. A., Best, A., and Crawford, I. L.: Refined low frequency active suspension, in: Proceedings of IMechE International Conference, 285-300, 1993.

Youn, I. and Hac, A.: Semi-active suspension with adaptive capability, J. Sound Vib., 180, 475-492, 1995.

Zin, A., Sename, O., Basset, M., Dugard, L., and Gissinger, G.: A nonlinear vehicle bicycle model for suspension and handling control studies, in: Proceedings of the IFAC Conference on Advances in Vehicle Control and Safety (AVCS), Genova, Italy, 638-643, 2004. 